\title{
Study on Fault Waveform Feature Extraction of Engine Ignition System based on Wavelet Analysis
}

\author{
Wang Fengjun \\ Wuxi Vocational Institute of Commerce, Wuxi, Jiangsu 214153, China
}

Keywords: Wavelet, Fault, Waveform, Feature extraction

\begin{abstract}
From the perspective of the application of signal feature extraction in the field of fault diagnosis, the method of signal feature extraction based on wavelet singularity is discussed in this paper. The theory of signal singularity detection based on a maximum value of wavelet transform modulus is discussed, the completeness of waveform feature extraction based on a maximum value of wavelet transform modulus is expounded, and finally, an analysis example of standard primary voltage waveform was given out.
\end{abstract}

\section{Introduction}

In case that some parts of the engine ignition system are abnormal, the structure of the primary voltage waveform signals in the state of the ignition system will change, representing the singularity of the signals, the singular points of the waveform signals and the irregular abrupt changes usually carry many important information of the signals, which is one of the important features of the signals. In the field of fault diagnosis, the abrupt change points of the signals often contain very abundant fault information, and they reflect the impact, vibration, friction, sudden speed change, structure deformation and fracture caused by fault; how to extract the information on signal characterization from abrupt change points of signals will be directly related to the fault diagnosis of the next step, thus to judge the time for singular points in waveform signals, and to realize quantitative description of the signal singularity, which has important significance in the fault diagnosis of the engine ignition system.

Fourier analysis once became a main tool for signal singularity analysis, but Fourier transformation lacks spatial locality, it can only determine the overall nature of signal singularity, and it is difficult to determine the location and distribution of singularities in space. We know that wavelet transform has the property of spatial localization. Therefore, it is effective to analyze the signal singularity and extract the features of waveform signals by use of wavelet transform.

In this paper, the feature extraction method based on the maximum value of wavelet transform modulus was preliminarily discussed from the application of signal analysis and processing in the field of fault diagnosis. This paper focuses on the discussion of application, but pays little attention to the mathematical derivation and proof.

\section{Wavelet Analysis and Signal Singularity Detection}

\subsection{Wavelet Function and Wavelet Transform.}

Suppose $\psi(x) \in L^{2}(R), L^{2}(R)$ represents the square integrable real number space, namely the signal space with limited energy, and its Fourier transform is $\bar{\psi}(\omega)$. When $\bar{\psi}(\omega)$ meets the permissive condition

$$
C_{\psi}=\int_{R} \frac{|\bar{\psi}(\omega)|^{2}}{|\omega|} \mathrm{d} \omega<\infty
$$

$\psi(x)$ is referred to as a basic or parent wavelet. After stretching and translating the parent 
wavelet, a wavelet sequence can be obtained. For any real number pair $(a, b)$, call the following function:

$$
\psi_{a, b}(x)=\frac{1}{\sqrt{|a|}} \psi\left[\frac{x-b}{a}\right] \quad a, b \in R \text { and } a \neq 0
$$

The continuous wavelet function which is generated from basic wavelet and depends on the parameters $(a, b)$, is called wavelet for short, when changing $a$, the waveform of the function can extend or compress along the time axis; when changing $b$, the waveform of the function can move along the time axis, so it is called scale factor, or position factor. If changing the parameters $a$ and $b$, a cluster of wavelet functions can form, then the signal $f(x)$ to be analyzed is decomposed according to the cluster function, and according to the expansion coefficient, we can know what is the signal component of the signal $f(x)$ at a certain local frequency band in a certain local time, so as to realize the time-frequency local analysis of the adjustable window.

For any function or signal $f(x)$, its continuous wavelet transform is defined as:

$$
W_{f}(a, b)=\int_{R} f(x) \bar{\psi}_{a, b}(x) \mathrm{d} x=\frac{1}{\sqrt{|a|}} \int_{R} f(x) \bar{\psi}\left[\frac{x-b}{a}\right] \mathrm{d} x
$$

Therefore, for any function $f(x)$, its wavelet transform is a binary function. This is very different from the Fourier transform.

Sometimes, for the sake of mathematical convenience, the following definition is often adopted, and the following transformation $W_{f}(s, x)$ is defined as the wavelet transform of $f \in L^{p}(R)$ :

$$
W_{f}(s, x)=f^{*} \psi_{s}(x)=\frac{1}{s} \int_{R} f(t) \psi\left[\frac{x-t}{s}\right] \mathrm{dt}
$$

Wherein $\psi_{s}(x)=\frac{1}{s} \psi\left(\frac{x}{s}\right)$, mainly showing that a different sum of expansion coefficient replaces the correlation with convolution.

Discretization is necessary for both the feasibility consideration of numerical calculation and the simplicity of theoretical analysis. The wavelet transform of discretized signal is usually taken as binary discrete, and the scale parameter is $2^{j}(f \in Z)$ and the form of discrete binary wavelet transform can be written as.

$$
W_{f}\left(2^{j}, x\right)=\frac{1}{2^{j}} \int_{R} f(t) \psi\left(\frac{x-t}{2^{j}}\right) \mathrm{dt}
$$

\subsection{Relationship Between the Maximum Value of Wavelet Transform Modulus and the Signal Singularity.}

Let $f(x) \in L^{2}(R),[a, b]$ is the closed interval of $R$, in case of $0<a<1, \forall \varepsilon>0$, the sufficient and necessary condition for the function $f(x)$ that is consistent Lipschitz exponent $a$ in $(a+\varepsilon, b$ $-\varepsilon)$ is as follows: if and only if there is a constant $A$ and $x \in(a+\varepsilon, b-\varepsilon)$, for $\forall a>0$.

$$
\left|W_{f}(s, x)\right| \leq A s^{a}
$$

On the scale $s$, in case of $x=x_{0}$, the following is true.

$$
\frac{\partial W_{f}(s, x)}{\partial x}=0
$$


$x_{0}$ is called the local extreme point of wavelet transform.

For $\forall \delta>0, \exists\left|x-x_{0}\right|<\delta$, the following formula is established.

$$
\left|W_{f}(s, x)\right| \leq\left|W_{f}\left(s, x_{0}\right)\right|
$$

$x_{0}$ is called the maximum value point of wavelet transform modulus. $W_{f}\left(s, x_{0}\right)$ is called the maximum value of wavelet transform modulus.

Therefore, for a certain point $x$ in the neighborhood $x_{0}$, the equation (6) indicates that as the scale $s$ approaches zero, $\left|W_{f}(s, x)\right|=O\left(s^{a}\right)$. For the singular point $x_{0}$, since its Lipschitz exponent is less than the Lipschitz exponent of the rest points in the neighborhood, when $s$ is sufficiently close to zero, the absolute value of the wavelet transform at $x_{0}$ attenuates in the slowest manner, and thus, the point $x$ in the neighborhood $x_{0}$ converges to $x_{0}$ and becomes the point of maximum modulus. $\left|W_{f}(s, x)\right|$ converges to $\left|W_{f}\left(s, x_{0}\right)\right|$ and $\left|W_{f}\left(s, x_{0}\right)\right|$ becomes the maximum value of modulus; as $0<a<1$, as the scale increases, the magnitude of this maximum value increases steadily, or remains basically the same. The maximum value point of the corresponding signal singular point can be propagated along the scale. Therefore, a wavelet transform modulus maxima representation of the signal can be obtained by using this local singularity.

When determining the singular position and singularity of the signal (namely the Lipschitz exponent) $a$, the wavelet transform is usually first discretized.

Set the maximum value of a certain modulus $\left|W_{f}\left(2^{j}, x_{0}\right)\right|$ on the scale $j$, take the logarithm of base 2 from both sides of the equation 6 , and then get the following formula.

$$
\log _{2}\left|W_{f}\left(2^{j}, x\right)\right| \leq \log _{2}(A)+j a
$$

The above formula is a measure of the overall singularity of the signal. When planning to test the singularity of a signal point, $x_{0}$ is just replaced by $x$.

\section{Waveform Feature Extraction and its Completeness}

The primary ignition waveform signals of automobile engine contain abundant fault diagnosis information. When a portion of the engine ignition system fails, the corresponding wave form is often caused to change in the time domain waveform, and signal singularity on the change points will inevitably change, so the singularity index should be used to distinguish the fault; the singularity of waveform signal can be depicted by the modulus maximum point of wavelet transform and therefore, a very natural idea is to use the modulus maximum point of waveform signal wavelet transform as a signal feature description, but the other data except the maximum modulus in binary wavelet transform are ignored. The question is how much information on the original waveform signal is contained at the maximum modulus point of the binary wavelet transform? Can the original signal be represented stably only from the information on the maximum modulus point of the binary wavelet transform? Mayer studied the question, proving that the maximum modulus point of wavelet transform failed to provide complete signal, and thus, he constructed different functions of wavelet transform with the same local modulus maxima on different scales; as a result, these functions only contain errors in the high frequency part. Therefore, Mayer's research shows that the maximum modulus point of wavelet transform effectively expresses the features reflected by the medium and low frequency components of original signals, and this expression is stable in terms of numerical value, so the features of the signals are mainly manifested in regular medium and low frequency signals, the signal features can be described by the modulus maxima of wavelet transform and the completeness is guaranteed.

In this paper, the structural characteristics of the primary voltage waveform of the engine ignition system are described by using the maximum modulus value of wavelet transform, and the 
characteristic values of fault symptoms are obtained and therefore, whether the primary voltage waveform of the ignition system can be reconstructed by its low frequency coefficient should be examined first. Figure 1 shows the low-frequency reconstruction waveform of the primary ignition waveform, the first image shows the original waveform of the primary ignition voltage, and the second image shows the waveform reconstruction by low frequency coefficient extracted from discrete wavelet decomposition coefficient of the original waveform.

In Figure 1, Figure (a) shows the original waveform, while Figure (b) is the waveform reconstructed by using the low-frequency coefficient of discrete wavelet decomposition. The maximum error between the original waveform and the reconstructed waveform is 8.532e-14. It can be known that the characteristics of waveform signals are mainly concentrated in medium and low frequency, and the characteristics of waveform signals can be completely characterized by the maximum modulus value of its wavelet transform and its completeness can be guaranteed.

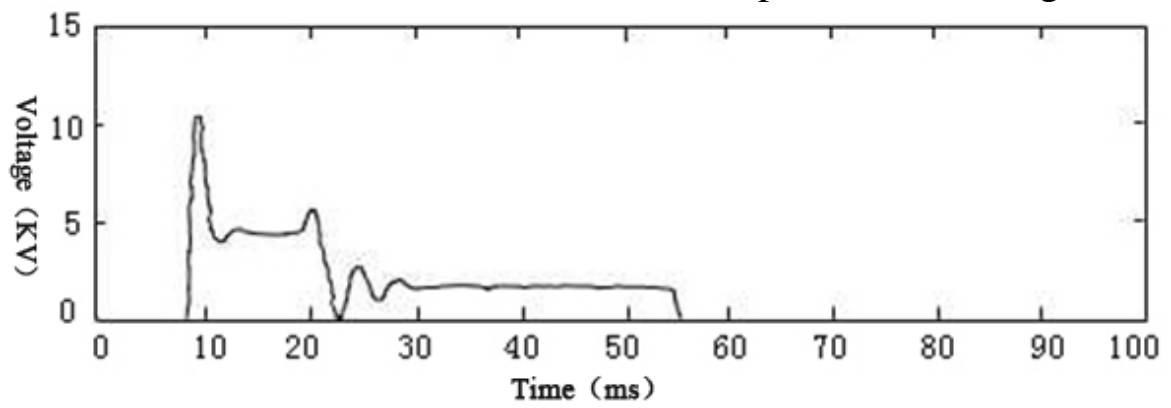

(a) Original waveform signal

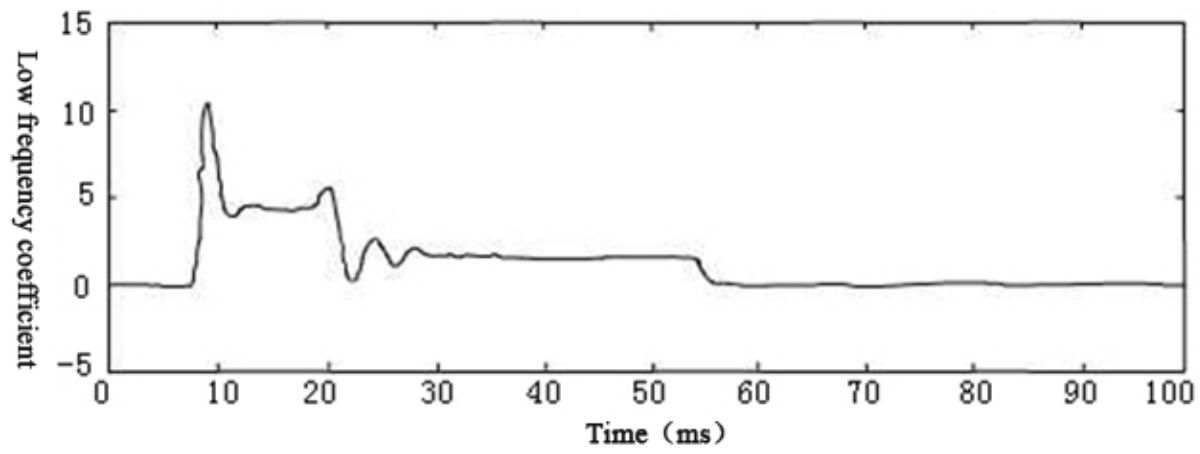

(b) Low-frequency reconstructed waveform signal

Figure 1 Waveform reconstructed by low frequency coefficient

\section{Feature Extraction of Primary Waveform of Ignition System}

\subsection{Primary Waveform of Ignition System.}

Ignition waveform is classified into primary voltage waveform and secondary voltage waveform. As the high voltage generated in the secondary circuit during the engine ignition will cause serious electromagnetic interference to the waveform signal acquisition instrument, the waveform signal of the primary voltage of the ignition system is collected in this subject. Figure 2 shows the standard primary ignition waveform, which depicts the time-varying waveform of the primary voltage in the process which the breaker contact is opened, closed and again opened.

Section A, spark plug arcing area, the waveform of the area, where the performance of spark plug can be observed; after opening the distributor, the primary circuit current drops rapidly, and through the ignition coil, high voltage is inducted in the secondary coil and the spark plug gap is punctured, so that arc discharge is generated from combustible gas in the spark plug gap, the high ignition voltage in the secondary circuit drops quickly down, and a certain voltage required by the spark plug electrode discharge is maintained to burn the mixture in the cylinder rapidly. The characteristic parameters of the section of waveform are high ignition voltage, spark duration and spark voltage. 


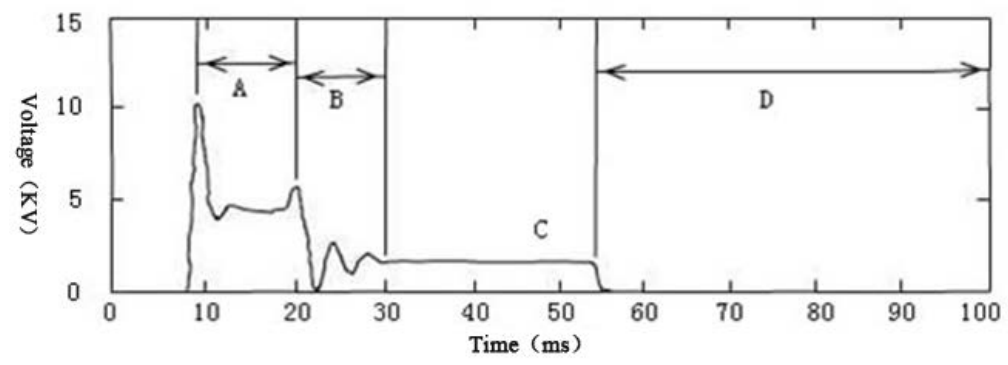

Figure 2 Standard primary voltage waveform

Section $\mathrm{B}$, the damping vibration area after arcing, represents the performance of the ignition coil and capacitor.

Point C, refers to a closed point of contact, and there is no current in the primary circuit when the contact begins to close. The position of the point on the horizontal axis indicates the size of the closed angle of contact and reflects the contact gap.

Section D, closed section of contact, reflects the status of the distributor.

The above is the meaning of each part of the waveform. According to the above description, it can be seen that the waveform contains the fault information of many parts of the ignition system, and fault diagnosis can be made by using the characteristic values extracted from the waveform.

\subsection{Feature Extraction of Waveform Signals.}
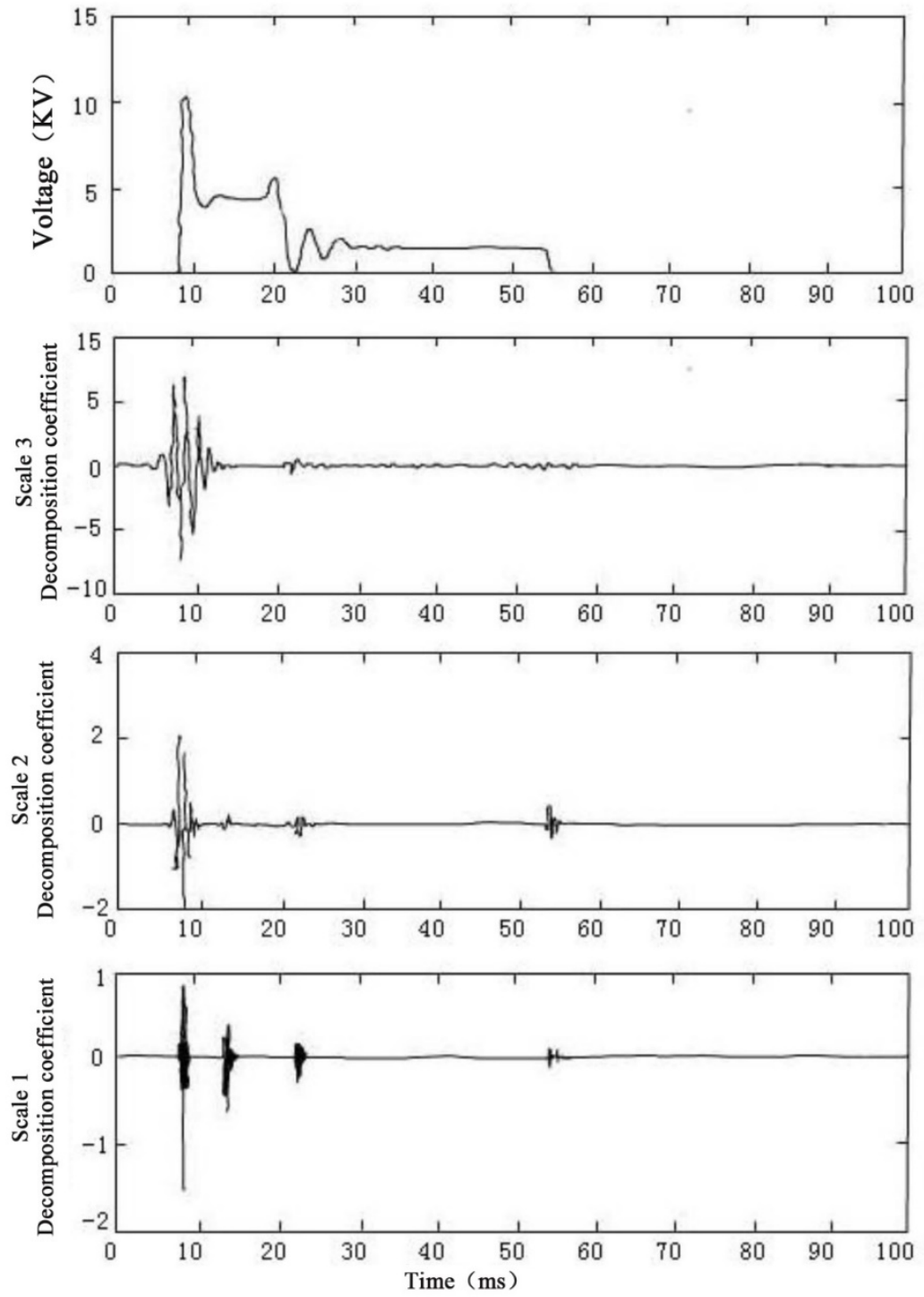

Figure 3 Multiscale analysis of waveform 
In a sense, feature extraction can be said to be a bottleneck in current research on mechanical fault diagnosis, and it is directly related to the accuracy of fault diagnosis and the reliability of early fault prediction. The maximum modulus value of the signal wavelet transform can be very approximate to the original signals. Therefore, features can be extracted from the ignition waveforms by use of the maximum modulus value of wavelet transform.

Figure 3 shows the results of multi-scale wavelet analysis on the standard primary ignition waveform corresponding to Figure 2.

In the multi-scale analysis of Figure 3, the selected wavelet function is db10 wavelet in Daubechies wavelet system. First of all, db10 wavelet is used for multi-scale wavelet decomposition of the waveform signals. The scale 6 is selected, then a low-frequency coefficient is extracted from the wavelet decomposition structure, and a wavelet reconstruction is carried out on scales 1, 2 and 3. The four images in Figure 3 show the original waveforms from top to bottom, scale 1 reconstruction waveform, scale 2 reconstruction waveform and scale 3 reconstruction waveform. It can be seen from the results that the waveform reconstructed by scale 1 is more effective than the waveforms reconstructed by other scales in testing the singularities of waveform signals. Therefore, the scale 1 waveform is selected as the waveform to be analyzed.

By analyzing the working principle and fault of the automobile engine ignition system and comparing the primary ignition waveform, the following six characteristic values are determined for fault diagnosis of the ignition system.

Ignition high pressure: the high pressure at the ignition of the spark plug is corresponding to the highest point on the waveform.

Spark duration: spark duration during ignition is corresponding to the period of time on the waveform from ignition high pressure to the end of spark pressure.

Closed angle: the angle at which the contact is closed is corresponding to the time at which the voltage falls from the battery to zero on the waveform.

The closed section has clutter: refers to whether there is a small voltage value in the closed stage of contact, that is, when the voltage is zero.

Overlap angle: refers to the difference of closed angle of each cylinder.

Oscillation frequency: refers to the oscillation when the spark voltage begins to drop. It is measured by the duration of oscillations on the waveform.

For the standard primary ignition waveform, the data of each characteristic value is shown in Table 1.

Table 1 Characteristic values of the standard waveform

\begin{tabular}{c|c}
\hline Name & Characteristic value \\
\hline High ignition voltage $\left[X_{1}\right]$ & 106.28 \\
\hline Spark time $\left[X_{2}\right]$ & 128 \\
\hline Closed angle $\left[X_{3}\right]$ & 553 \\
\hline The closed segment has clutter $\left[X_{4}\right]$ & 0 \\
\hline Overlap angle $\left[X_{5}\right]$ & 0 \\
\hline Oscillation frequency $\left[X_{6}\right]$ & 160 \\
\hline
\end{tabular}

Table 1 shows the characteristic values extracted from the standard primary ignition waveforms after wavelet analysis. The six characteristic values have no units, and they are obtained by the abscissa operations of the singular points shown in the wavelet multi-resolution graphs by use of standard waveforms in scale 1 . Because these data are required to be input to the established neural network diagnostic system and are to be preprocessed, their units will be removed after preprocessing. Therefore, the characteristic values of the waveforms can be expressed in this simple way without affecting subsequent operations. 


\section{Conclusion}

In this paper, the method of signal feature extraction based on wavelet singularity is discussed from the perspective of the application of signal feature extraction in the field of fault diagnosis. First, the definition of signal singularity and the basic concept of wavelet transform are summarized, then the theory of signal singularity detection based on the maximum modulus value of wavelet transform is discussed, the completeness of waveform feature extraction based on the maximum modulus value of wavelet transform is discussed, and finally, the analysis example of standard primary voltage waveforms is given out.

\section{References}

[1] Tan Xingguo, Li Qingmin, Wang Hui. Advances and trends of energy storage technology in Microgrid [J].International Journal of Electrical Power \& Energy Systems, 2013, 44(1): 179-191.

[2] Chen Z, Ding M, Su J. Modeling and control for largecapacity battery energy storage system [C]//ElectricUtility Deregulation and Restructuring and PowerTechnologies (DRPT), 2011: 1429-1436.

[3] Syed F U, Kuang Mingl, Smith M, et al. FuzzyGain-Scheduling Proportional-Integral control for improving engine power and speed behavior in a hybrid electric vehicle [J]. IEEE Transactions on Vehicular Technology, 2009, 58(1): 69-84.

[4] Li Xiangjun, Song Yujin, Han SB. Frequency control in micro-grid power system combined with electrolyzer system and fuzzy PI controller [J]. Journal of Power Sources, 2008, 180(1): 468-475.

[5] Dannehl J, Wessels C, Fuchs F W. Limitations of Voltage-Oriented PI current control of Grid-Connected PWM rectifiers with LCL filters [J]. IEEE Transactions on Industrial Electronics, 2009, 56(2): 380-388. 